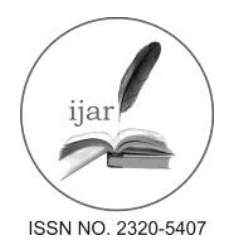

Journal homepage: http://www.journalijar.com

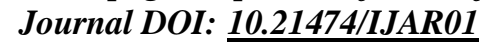

INTERNATIONAL JOURNAL

OF ADVANCED RESEARCH

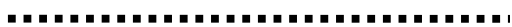

\title{
MILD AND MODERATE OBESITY REMAINS THE SOLE CAUSE FOR RISE IN HS-CRP, TNF $\alpha$ AND LEPTIN IN OBESE INDIVIDUALS IN SPITE OF PERFORMING MODERATE ACTIVITY AND CONSUMING ANTIOXIDANT AND PREBIOTIC RICH FOODS FREQUENTLY - A CROSS SECTIONAL STUDY IN URBAN VADODARA.
}

${ }^{*}$ Mini Sheth ${ }^{1}$, Jyoti Prakash ${ }^{1}$, Swati Parnami ${ }^{1}$, Sania Quraishi ${ }^{1}$, Anil Kumar ${ }^{2}$, Manish Jain ${ }^{2}$ and Manoj Gote ${ }^{2}$.

1. Department of Food and Nutrition, Faculty of Family and Community Sciences, The Maharaja Sayajirao University of Baroda, Vadodara.

2. Innovation centre TATA Chemicals Ltd., Pune, Maharashtra.

\section{Manuscript Info}

Manuscript History:

Received: 18 March 2016

Final Accepted: 22 April 2016

Published Online: May 2016

Key words:

*Corresponding Author

Mini Sheth.

\begin{abstract}
Background:- Obesity has reached pandemic proportions globally leading to various co-morbidities and the risk factors contributing to these morbidities need to be researched more intensively.
\end{abstract}

Objectives:- To determine the various factors contributing to levels of inflammatory markers in obese individuals and compare with non obese subjects.

Method:- Using a cross sectional study design, from a total of 252 subjects, 167 obese and 87 non obese subjects living in urban Vadodara, aged 20-50 years, were studied for their BMI $(\mathrm{kg} / \mathrm{m} 2)$ and physical activity level, serum hs-CRP levels (measured by nephelometry) TNF $\alpha$ and leptin levels (analyzed using Milliplex Map, Human Metabolic Hormone Magnetic Bead Panel (HMHAG). Frequency of intake of antioxidant and prebiotic rich foods was measured using a food frequency questionnaire and fecal Bifidobacteria was determined using standard methods.

Results:- The mean BMI of the subjects was 28.6 and $21.39 \mathrm{~kg} / \mathrm{m}^{2}$ respectively whereas the mean physical activity levels were 694 and 717 MET mins/week for obese and non obese respectively. ANOVA test revealed a significant linear rise in the hs-CRP, TNF $\alpha$ and leptin levels with increase in obesity. Hs-crp was positively and significantly correlated with physical activity and negatively correlated with Bifidobacteria colonization in the gut. No significant correlation was found between the frequency of intake of antioxidant and prebiotic rich foods and all inflammatory markers.

Conclusion:- Moderate obesity stands as a major cause for rise in the inflammatory markers followed by poor colonization of Bifidobacteria in the Gut. Moderate activity and increased intake of antioxidant rich foods does not lower the hs-CRP, TNF $\alpha$ and leptin significantly.

Copy Right,IJAR, 2016...All rights reseryed...

\section{Introduction:-}

In the past 50 years, the occurrence of human obesity has risen tremendously across the globe. High-income countries are not the only ones affected by obesity; the condition is on an alarming rise in the developing world as well. Globally one in six adults are obese and nearly 2.8 million individuals die each year due to overweight or 
obesity (WHO 2012). Obesity has been associated with a number of other metabolic disorders characterized by chronic, systemic, low-grade inflammation. Reframing obesity as an inflammatory condition has had a wide impact on our conceptualization of obesity-associated diseases. Despite no overt infection or auto-antigen mediated immune cell activation, chronic inflammation during obesity is an established instigator of several diseases like the type 2 diabetes, defective immunity, atherosclerosis, certain cancers, CNS dysfunction and dementia (Dixit VD,2008; Hotamisligil GS, 2008; Luchsinger JA, 2009; Yang H, et al. 2009)

Elevated serum levels of C-reactive protein (CRP) have been widely considered to be nonspecific but sensitive markers of the acute inflammatory response. CRP, as an acute-phase protein which originates in the liver as well as adipose tissue, has many pathophysiologic roles in low grade inflammation in obesity (Wu T, 2010).

Tumor necrosis factor (TNF, tumor necrosis factor alpha, TNF $\alpha$, cachexin, or cachectin) is a cell signaling protein (cytokine) involved in systemic inflammation and is one of the cytokines that make up the acute phase reaction. Studies have shown that an array of inflammatory cytokines are increased in obese tissues, including interleukin TNF $\alpha$,(IL)-6, IL-1 $\beta$, CCL2, and others (Shoelson S, 2006).

Therefore an attempt was made to establish the inflammatory link of obesity with respect to some of the inflammtory biomarkers viz. hsCRP, TNF $\alpha$ and leptin.

\section{Methods and materials:- \\ Location of the study:-}

The urban area of Vadodara, Gujarat was conveniently selected to conduct the research study. The subjects were taken from free living population from yoga centers, gyms, educational institutes, hostels and gardens

\section{Study Protocol:-}

Two fifty two adults of the age group of 20-50 years were screened for BMI and physical activity pattern using purposive sampling. Out of 252 subjects 85 non obese and 167 obese subjects were enrolled and studied for their background information, family history and medical history, physical activity pattern using GPAQ (WHO,2007), dietary intake of prebiotic and antioxidant rich foods. The inclusion criteria were patients of either sex between age group 20-50 yrs, suffering from obesity with BMI >25 and the exclusion criteria were patients suffering from diabetes, hypertension, CVD, celiac disease , HIV, cancer, renal disorder and liver disorder, infections and inflammatory disorders, hospitalization since last one month.

Blood samples were collected for hs-CRP, TNF, leptin and gut microbial counts in terms of fecal bifidobacteria.

\section{Laboratory Procedures:-}

Venous blood samples were taken from all subjects at baseline which was allowed to clot for at least 30 minutes before centrifugation for 10 minutes at $1000 \mathrm{xg}$ and then serum was removed and store samples at $-20^{\circ} \mathrm{C}$. Serum concentrations of CRP were measured using Nephelometry technology, where during reaction, antigen-antibody complexes are generated in solution which is then quantified with the aid of a light beam. The light when incident on the immune complex, is scattered which is captured by lens fit at right angles to the incident light. This scattered light is then measured to determine concentration of the analyte of interest. The analyzer which works under this principle is BN II [Siemens].

Determination of TNFa and Leptin: These were determined using HMHMAG-34K- MILLIPLEX MAP metabolic Hormone magnetic bead Panel. 5 kits of HMHMAG-34K were procured from Mehta Sales Chemi Pvt. Ltd Vadodara. $200 \mu \mathrm{L}$ of Assay Buffer was added into each well of the plate, sealed and mixed for 10 minutes at room temperature $\left(20-25^{\circ} \mathrm{C}\right)$. After decanting, assay Buffer, $(25 \mu \mathrm{L})$ was added to each standard solution and samples in the well followed by addition of $25 \mu \mathrm{L}$ Beads to each well. The wells were sealed and incubated overnight at $4^{\circ} \mathrm{C}$. The contents were removed and the wells were washed $3 \mathrm{X}$ with $200 \mu \mathrm{L}$ wash buffer followed by addition of $50 \mu \mathrm{L}$ of Detection Antibodies into each well; Incubated for 1 hour at room temperature $\left(20-25^{\circ} \mathrm{C}\right)$ and then addition of $50 \mu \mathrm{L}$ of Streptavidin-Phycoerythrin was made to each well containing the $50 \mu \mathrm{L}$ of Detection Antibodies; Incubated for 30 minutes. all the well contentswere removed and washed $3 X$ with $200 \mu \mathrm{L}$ Wash Buffer.

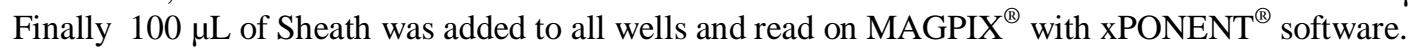

Determination of Bifidobacteria: $1 \mathrm{~g}$ of thawed fecal sample was accurately weighed, homogenized with diluent in a stomacher, serially diluted up to $10^{10}$ dilutions. $0.1 \mathrm{ml}$ was plated using surface plate technique and selective 
bifidobacteria medium was added, allowed to set and the petridishes were inverted in an anaerobic jar with gas packs. After 48 hour of incubation at $37^{\circ}$ celcius, the colonies developed were counted using a colony counter. ( FAO/WHO 1979; hi media manual, Mumbai 2011).

\section{Results:-}

General information of non-obese and obese subjects:-

As seen in table 1 most of the non-obese and the obese subjects were females who were highly educated and belonged to the age group of 20-35 years irrespective of their activity levels.

Table 1:- Background information of non-obese and obese subjects

\begin{tabular}{|l|l|l|l|}
\hline \multicolumn{2}{|l|}{ Parameters } & $\begin{array}{l}\text { Non-obese } \\
\mathbf{N = 8 5}(\boldsymbol{\%})\end{array}$ & $\begin{array}{l}\text { Obese } \\
\mathbf{N = 1 6 7}(\boldsymbol{\%})\end{array}$ \\
\hline \multirow{3}{*}{ Age } & $\mathbf{2 0 - 3 5}$ yrs & $57(67.1)$ & $92(55.1)$ \\
\cline { 2 - 4 } & $\mathbf{3 6 - 5 0}$ yrs & $28(32.9)$ & $75(44.9)$ \\
\hline \multirow{3}{*}{ Gender } & Male & $17(20)$ & $41(24.6)$ \\
\cline { 2 - 4 } & Female & $68(80)$ & $126(75.4)$ \\
\hline \multirow{5}{*}{ Education } & Diploma & $23(27.1)$ & $44(26.3)$ \\
\cline { 2 - 4 } & Graduate & $6(7.1)$ & $9(5.4)$ \\
\cline { 2 - 4 } & Post graduate & $46(54.1)$ & $90(53.9)$ \\
\cline { 2 - 4 } & Higher studies & $6(7.1)$ & $24(14.4)$ \\
\hline
\end{tabular}

Note: Figures in parenthesis represents percent of subjects

As seen in table 2 the family history for the NCDs revealed a higher prevalence of obesity, diabetes and hypertension in the obese subjects when compared to the non-obese subjects and this difference was not found to be statistically significant $(\mathrm{p}<0.001)$.

Table 2:- Family history of non-obese and obese subjects.

\begin{tabular}{|l|l|l|l|l|}
\hline $\begin{array}{l}\text { Family History } \\
\begin{array}{l}\text { Both parents, single parents and } \\
\text { siblings) }\end{array}\end{array}$ & $\begin{array}{l}\text { Non-obese } \\
\mathbf{n = 8 5}(\boldsymbol{\%}) \\
\mathbf{n = 1 6 7}(\boldsymbol{\%})\end{array}$ & $\chi^{2}$ value & $p$-value \\
\hline Obesity & $20(23.52)$ & $43(25.74)$ & $\mathbf{0 . 1 4}^{\mathrm{NS}}$ & $\mathbf{0 . 7 0 1}$ \\
\hline Diabetes Mellitus & $40(47.05)$ & $98(58.68)$ & $\mathbf{3 . 0 6}^{\mathrm{NS}}$ & $\mathbf{0 . 0 8 0}$ \\
\hline Hypertension & $14(16.47)$ & $46(27.54)$ & $\mathbf{3 . 7 9}^{\mathrm{NS}}$ & $\mathbf{0 . 0 5 1}$ \\
\hline
\end{tabular}

Note: Figures in parenthesis represents percent of subjects

Most subjects in both non-obese and obese categories were not suffering from any dental problems [Table 3(a)] and very small percentage of the subjects in both obese and non-obese categories suffered from gastrointestinal problems frequently (Table 3(b))

Table 3:- Immediate medical history of the obese and non-obese subjects with regards to their dental problems and GI problems.

(a) Dental problems of non-obese and obese subjects

\begin{tabular}{|c|c|c|}
\hline Dental problems & Non-obese $(\mathbf{n}=\mathbf{8 5})(\boldsymbol{\%})$ & Obese $(\mathbf{n}=\mathbf{1 6 7})(\boldsymbol{\%})$ \\
\hline Gum disease & ----- & $2(1.2)$ \\
\hline Cavities & $4(7)$ & $24(11.9)$ \\
\hline None & $81(93)$ & $141(86.9)$ \\
\hline
\end{tabular}

(b) Frequency of occurrence of Gastrointestinal problems in non-obese and obese subjects.

\begin{tabular}{|l|l|l|l|l|l|l|}
\hline GI problems & Non-obese $(\mathbf{n}=85)(\%)$ & \multicolumn{3}{l|}{ Obese $(\mathbf{n = 1 6 7 )} \mathbf{( \% )}$} \\
\hline & Never & $\begin{array}{l}\text { Less } \\
\text { frequently }\end{array}$ & $\begin{array}{l}\text { More } \\
\text { frequently }\end{array}$ & Never & $\begin{array}{l}\text { Less } \\
\text { frequently }\end{array}$ & $\begin{array}{l}\text { More } \\
\text { frequently }\end{array}$ \\
\hline Nausea & $76(89.41)$ & $9(10.58)$ & --- & $138(82.63)$ & $28(16.76)$ & $1(0.59)$ \\
\hline Heart burn & $84(98.82)$ & $1(1.17)$ & ---- & $150(89.82)$ & $15(8.98)$ & $2(1.19)$ \\
\hline Abdominal pain & $63(74.11)$ & $21(24.70)$ & $1(1.17)$ & $119(71.25)$ & $47(28.14)$ & $1(0.59)$ \\
\hline Constipation & $63(74.11)$ & $19(22.35)$ & $3(2.52)$ & $116(69.46)$ & $38(22.75)$ & $13(7.78)$ \\
\hline Flatulence & $82(96.47)$ & $2(2.35)$ & $1(1.17)$ & $145(86.82)$ & $13(7.78)$ & $9(5.38)$ \\
\hline Diarrhoea & $71(83.52)$ & $13(15.29)$ & $1(1.17)$ & $140(83.83)$ & $26(15.56)$ & $1(0.59)$ \\
\hline Dysentery & $81(95.29)$ & $4(4.70)$ & ---- & $154(92.21)$ & $13(7.78)$ & ---- \\
\hline
\end{tabular}


Table 4 revealed that the non-obese subjects $(74 \%)$ and the obese subjects $(54 \%)$ controlled the weight by dietary management.

Table 4:- Measures taken to control weight by non-obese and obese subjects.

\begin{tabular}{|c|c|c|c|}
\hline & Non-obese $(\mathbf{n}=\mathbf{8 5}) \mathbf{( \% )}$ & Obese $(\mathbf{n}=\mathbf{1 6 7}) \mathbf{( \% )}$ & $\chi^{2}$ value $(\boldsymbol{p}$-value $)$ \\
\hline Controlling weight & $63(74.11)$ & $113(67.66)$ & $1.10^{\mathrm{NS}}(0.29)$ \\
\hline Dietary supplement & ---- & $6(3.59)$ & $9.09 * *(0.002)$ \\
\hline Dietary management & $63(74.11)$ & $91(54.49)$ & $6.27 *(0.012)$ \\
\hline Controlling sugar & $44(51.76)$ & $59(35.32)$ & $0.58^{\mathrm{NS}}(0.44)$ \\
\hline Controlling CHO & $23(27.05)$ & $53(31.73)$ & $1.30^{\mathrm{NS}}(0.252)$ \\
\hline Controlling fats/oil & $40(47.05)$ & $66(39.52)$ & - \\
\hline
\end{tabular}

As revealed in the table 5, the mean BMI of the obese subjects was $28.56 \mathrm{~kg} / \mathrm{m}^{2}$ indicating that they belonged to grade I obesity and no significant difference was observed in their mean BMI values with respect to their activity levels. There was no significant difference observed in the activity level and consumption of prebiotic rich foods in the obese and non-obese subjects, however the obese subjects consumed significantly higher amount of anti oxidant rich foods.

All the inflammatory markers viz. hs-CRP, TNF $\alpha$ and leptin were significantly higher in obese subjects as compared to the non-obese subject.

Table 5:- Mean values for BMI, physical activity level, the inflammatory markers, intake of antioxidants, and probiotic and prebiotic rich foods, Bifidobacteria counts in non-obese and obese subjects (Mean \pm SD)

\begin{tabular}{|c|c|c|c|}
\hline Parameters & $\begin{array}{l}\text { Total Non-obese } \\
(n=85)\end{array}$ & $\begin{array}{l}\text { Total Obese } \\
(n=167)\end{array}$ & $\begin{array}{l}\text { 't' value } \\
\text { (p value) }\end{array}$ \\
\hline Height (cms) & $160.47 \pm 7.91$ & $158.58 \pm 7.79$ & $1.809^{\mathrm{NS}}(\mathrm{p}-0.072)$ \\
\hline Weight (cms) & $55.44 \pm 6.63$ & $71.96 \pm 10.75$ & $12.957 * * *(\mathbf{p}-0.000)$ \\
\hline BMI $\left(\mathbf{k g} / \mathbf{m}^{2}\right)$ & $21.38 \pm 1.16$ & $28.56 \pm 2.98$ & $21.315 * * *(\mathbf{p}-0.000)$ \\
\hline MET Minutes & $717.88 \pm 727.45$ & $694.39 \pm 635.26$ & $0.262^{\mathrm{NS}}(p-0.793)$ \\
\hline \#Prebiotic and probiotic rich foods & $32.22 \pm 6.26$ & $31.98 \pm 5.84$ & $0.310^{\mathrm{NS}}(\mathrm{p}-0.757)$ \\
\hline *Antioxidant rich foods & $42.55 \pm 9.21$ & $45.17 \pm 10.05$ & $2.011 *(p-0.045)$ \\
\hline hs-CRP (mg/l) & $1.43 \pm 1.87$ & $2.91 \pm 2.69$ & $3.535 * *(p-0.001)$ \\
\hline LEPTIN (pg/ml) & $5165.72 \pm 3516.94$ & $12106.26 \pm 7226.25$ & $6.235 * * *(\mathbf{p}-0.000)$ \\
\hline TNF $\alpha(\mathrm{pg} / \mathrm{ml})$ & $3.87 \pm 2.05$ & $6.87 \pm 8.10$ & $2.515 *(p-0.013)$ \\
\hline
\end{tabular}

Note: ns=non-significant, $\mathrm{p}<0.05: *, \mathrm{p}<0.01:^{* *}, \mathrm{p}<0.001: * * * ; \#, *$ Frequency of Consumption

As seen in table 6 and 7 increasing age did not seem to have a negative influence on various inflammatory markers, BMI and physical activity levels. However female subjects significantly had higher hs-CRP and leptin levels than their male counterparts.

Table 6:- Mean values of various parameters based upon the age of the obese subjects (Mean \pm SD).

\begin{tabular}{|c|c|c|c|}
\hline Parameters & Age 20-35 yrs & Age 35-50 yrs & 't' value (p-value) \\
\hline BMI & $26.6 \pm 4.34$ & $27.4 \pm 3.55$ & $1.34^{\mathrm{NS}}(\mathrm{p}-0.180)$ \\
\hline MET & $635.58 \pm 595.79$ & $728.82 \pm 729.55$ & $1.011^{\mathrm{NS}}(p-0.310)$ \\
\hline $\begin{array}{l}* \text { Prebiotic and probiotic rich } \\
\text { foods }\end{array}$ & $31.6 \pm 5.72$ & $31.75 \pm 6.37$ & $0.17^{N S}(p-0.859)$ \\
\hline *Antioxidant rich foods & $45.36 \pm 8.57$ & $44.91 \pm 11.10$ & $0.33^{\mathrm{NS}}(\mathrm{p}-0.740)$ \\
\hline hs-CRP(mg/l) & $2.49 \pm 2.57$ & $2.72 \pm 2.66$ & $0.63^{\mathrm{NS}}(\mathrm{p}-0.524)$ \\
\hline Leptin (pg/mL) & $10048.96 \pm 7730.16$ & $11466.46 \pm 6234.14$ & $1.39^{\mathrm{NS}}(p-0.164)$ \\
\hline TNFa(pg/mL) & $5.86 \pm 7.38$ & $6.71 \pm 7.21$ & $0.82^{\mathrm{NS}}(\mathrm{p}-0.408)$ \\
\hline $\begin{array}{l}\text { FecalBifidobacteria } \\
\log _{10} \text { values }(\mathrm{CFU} / \mathrm{g})(\mathrm{n}=42)\end{array}$ & $5.62 \pm 0.35$ & $5.76 \pm 0.44$ & $0.933^{\mathrm{NS}}(\mathrm{p}-0.355)$ \\
\hline
\end{tabular}

NOTE:NS = NON-SIGNIFICANT; * Frequency of Consumption 
Table 7:- Mean values of various parameters based upon the gender of the obese subjects.

\begin{tabular}{|l|l|l|l|}
\hline Parameters & Male(49) & Female(162) & 't'value (p-value) \\
\hline BMI & $27.32 \pm 3.42$ & $26.85 \pm 4.23$ & $0.70^{\mathrm{NS}}(\mathrm{p}-0.484)$ \\
\hline MET & $764.73 \pm 742.15$ & $645.44 \pm 623.29$ & $1.12^{\mathrm{NS}}(\mathrm{p}-0.263)$ \\
\hline $\begin{array}{l}\text { \#Prebiotic and probiotic rich } \\
\text { foods }\end{array}$ & $32.24 \pm 6.24$ & $31.49 \pm 5.84$ & $0.77^{\mathrm{NS}}(\mathrm{p}-0.439)$ \\
\hline \#Antioxidant rich foods & $46.37 \pm 8.58$ & $44.81 \pm 9.94$ & $0.98^{\mathrm{NS}}(\mathrm{p}-0.325)$ \\
\hline hs-CRP (mg/l) & $1.76 \pm 1.35$ & $2.83 \pm 2.83$ & $2.55^{*}(\mathrm{p}-0.011)$ \\
\hline Leptin (pg/ml) & $6584.99 \pm 6150.42$ & $11812.45 \pm 7057.14$ & $4.63^{* * *}(\mathrm{p}-0.000)$ \\
\hline TNFa (pg/ml) & $6.27 \pm 2.81$ & $6.18 \pm 8.20$ & $0.06^{\mathrm{NS}}(\mathrm{p}-0.945)$ \\
\hline $\begin{array}{l}\text { Fecal Bifidobacteria } \\
\log _{\mathbf{1 0}} \text { (CFU/g) }\end{array}$ & 5.740 .45 & 5.630 .35 & $0.59^{\mathrm{NS}}(\mathrm{p}-0.553)$ \\
\hline
\end{tabular}

NOTE: NS= NON-SIGNIFICANT, P<0.05: *, P<0.001:***;\# Frequency of Consumption

Table 8 shows that the subjects who consumed prebiotic foods more frequently also consumed antioxidant rich foods more frequently.

Table 8:- Mean values of various parameters based upon the Frequency of consumption of Prebiotic rich foods by the obese subjects

\begin{tabular}{|l|l|l|l|}
\hline Parameters & $\begin{array}{l}\text { Less Frequent 16-30 } \\
\mathbf{( 8 3 )}\end{array}$ & $\begin{array}{l}\text { More Frequent 31-50 } \\
\mathbf{( 1 2 8 )}\end{array}$ & 't' value (p-value) \\
\hline BMI & $26.87 \pm 3.56$ & $27.02 \pm 4.35$ & $0.25^{\mathrm{NS}}(\mathrm{p}-0.797)$ \\
\hline MET & $540.04 \pm 601.22$ & $759.45 \pm 672.61$ & $2.41^{* *}(\mathrm{p}-0.017)$ \\
\hline$*$ Antioxidant rich foods & $41.88 \pm 8.59$ & $47.31 \pm 9.72$ & $4.14^{* * *}(\mathrm{p}-0.000)$ \\
\hline hs-CRP $(\mathbf{m g} / \mathbf{l})$ & $2.93 \pm 3.13$ & $2.36 \pm 2.17$ & $1.56^{\mathrm{NS}}(\mathrm{p}-0.120)$ \\
\hline Leptin $(\mathbf{p g} / \mathbf{m l})$ & $10827.17 \pm 6960.83$ & $10472.88 \pm 7360.28$ & $0.34^{\mathrm{NS}}(\mathrm{p}-0.729)$ \\
\hline TNF $\boldsymbol{\alpha}(\mathbf{p g} / \mathbf{m l})$ & $7.07 \pm 11.06$ & $5.64 \pm 2.92$ & $1.39^{\mathrm{NS}}(\mathrm{p}-0.165)$ \\
\hline $\begin{array}{l}\text { Fecal Bifidobacteria } \\
\log _{\mathbf{1 0}}(\mathbf{C F U} / \mathbf{g})\end{array}$ & 5.710 .39 & 5.600 .35 & $0.92^{\mathrm{NS}}(\mathrm{p}-0.361)$ \\
\hline
\end{tabular}

NOTE:NS =NON-SIGNIFICANT, $\mathrm{P}<0.01:^{* *}, \mathrm{P}<0.001: * * * * *$ Frequency of Consumption

Table 9 ( $a$ and $b$ ) reveals that as the grades of BMI increases the consumption of antioxidant rich foods increased significantly without significantly affecting the activity levels, prebiotic food consumption and Bifidobacteria levels. Also ANOVA test revealed that all the inflammatory markers increased significantly with increase in BMI.

Table 9:-

(a) ANOVA to study the effect of degree of obesity on various parameters in the obese and non obese subjects

\begin{tabular}{|c|c|c|c|c|c|c|c|}
\hline BMI range & $\begin{array}{l}\text { MET } \\
(\mathrm{n}=211)\end{array}$ & $\begin{array}{l}\text { \#Prebiotic } \\
\text { and } \\
\text { probiotic } \\
\text { rich foods } \\
(\mathbf{n}=\mathbf{2 1 1})\end{array}$ & $\begin{array}{l}\text { \#Antioxida } \\
\text { nt rich } \\
\text { foods } \\
\text { n=211) }\end{array}$ & $\begin{array}{l}\text { Bifido- } \\
\text { bacteria } \\
\log _{10} \\
(\mathrm{CFU} / \mathrm{g}) \\
(\mathrm{n}=\mathbf{4 4})\end{array}$ & $\begin{array}{l}\text { hsCRP } \\
(\mathrm{mg} / \mathrm{l}) \\
(\mathrm{n}=211)\end{array}$ & $\begin{array}{l}\text { Leptin } \\
(\mathbf{p g} / \mathrm{ml}) \\
(\mathbf{n}=209)\end{array}$ & $\begin{array}{l}\text { TNF } \\
(\mathbf{p g} / \mathrm{ml}) \\
(\mathbf{n}=211)\end{array}$ \\
\hline $18.5-22.9$ & $625.95 \pm 715.96$ & $31.32 \pm 6.55$ & $44.21 \pm 8.57$ & ----- & $1.43 \pm 1.87$ & $5165.72 \pm 3516.94$ & $3.87 \pm 2.05$ \\
\hline $25-27.9$ & $746.82 \pm 664.50$ & $31.56 \pm 5.66$ & $43.51 \pm 9.92$ & $5.68 \pm 0.39$ & $2.48 \pm 2.75$ & $10397 \pm 6626.05$ & $6.34 \pm 7.09$ \\
\hline $28-29.9$ & $654.19 \pm 590.61$ & $32.65 \pm 6.29$ & $47.67 \pm 10.55$ & $5.65 \pm 0.33$ & $3.23 \pm 2.37$ & $11746.78 \pm 6431.29$ & $6.89 \pm 2.97$ \\
\hline$>30$ & $587.84 \pm 619.03$ & $31.19 \pm 5.65$ & $47.27 \pm 8.50$ & $5.61 \pm 0.37$ & $3.53 \pm 2.79$ & $16402.39 \pm 7.811$ & $8.07 \pm 12.99$ \\
\hline $\begin{array}{l}\text { ANOVA } \\
\text { (p value) }\end{array}$ & $\begin{array}{l}0.658^{\mathrm{NS}} \\
(\mathrm{p}-0.579)\end{array}$ & $\begin{array}{l}0.525^{\mathrm{NS}} \\
(\mathrm{p}-0.665)\end{array}$ & $\begin{array}{l}2.589^{*} \\
(\mathrm{p}-0.054)\end{array}$ & $\begin{array}{l}0.145^{\mathrm{NS}} \\
(\mathrm{p}-0.865)\end{array}$ & $\begin{array}{l}6.018 * * \\
(\mathrm{p}-0.001)\end{array}$ & $\begin{array}{l}22.165^{* * *} \\
(\mathrm{p}-0.000)\end{array}$ & $\begin{array}{l}2.597^{*} \\
(\mathrm{p}-0.038)\end{array}$ \\
\hline
\end{tabular}

Note: $\mathrm{ns}=$ non-significant, $\mathrm{p}<0.05: *, \mathrm{p}<0.01:^{* *}, \mathrm{p}<0.001$ : $^{*} *$; $\#$ Frequency of Consumption 
(b) Post Hoc; LSD Test to study the effect of degree of obesity on various parameters in the obese\& non obese subject

\begin{tabular}{|c|c|c|c|c|c|c|c|}
\hline \multicolumn{8}{|c|}{ Post Hoc; LSD Test } \\
\hline \multirow[t]{2}{*}{ Dependent Variable } & \multirow{2}{*}{$\begin{array}{c}\text { BMI } \\
\text { Range }\end{array}$} & \multirow{2}{*}{$\begin{array}{c}\text { BMI } \\
\text { Range }\end{array}$} & \multirow{2}{*}{ Mean Difference } & \multirow[t]{2}{*}{ Std. Error } & \multirow[t]{2}{*}{ Sig. } & \multicolumn{2}{|c|}{ 95\% Confidence Interval } \\
\hline & & & & & & Lower Bound & Upper Bound \\
\hline \multirow[t]{3}{*}{ \#Antioxidant rich foods } & \multirow[t]{2}{*}{$25-27.9$} & 28-29.9 & $-4.163^{*}$ & 1.789 & .021 & -7.69 & -.64 \\
\hline & & $>\mathbf{3 0}$ & $-3.758^{*}$ & 1.883 & .047 & -7.47 & -.05 \\
\hline & $>\mathbf{3 0}$ & 25-27.9 & $3.758^{*}$ & 1.883 & .047 & .05 & 7.47 \\
\hline \multirow[t]{5}{*}{ hs-CRP } & \multirow[t]{3}{*}{ 18.5-22.9 } & 25-27.9 & $-1.051^{*}$ & 0.458 & .023 & -1.955 & -.147 \\
\hline & & 28-29.9 & $-1.802^{*}$ & 0.531 & .001 & -2.849 & -.7551 \\
\hline & & $>\mathbf{3 0}$ & $-2.096^{*}$ & 0.553 & .000 & -3.186 & -1.00 \\
\hline & \multirow[t]{2}{*}{ 25-27.9 } & 18.5-22.9 & $1.051^{*}$ & 0.458 & .023 & .1473 & 1.955 \\
\hline & & $>30$ & $-1.044^{*}$ & 0.496 & .037 & -2.024 & -.0656 \\
\hline \multirow[t]{5}{*}{ Leptin } & \multirow[t]{3}{*}{$18.5-22.9$} & $25-27.9$ & $-5232.210^{*}$ & 1162.81 & .000 & -7524.82 & -2939.59 \\
\hline & & 28-29.9 & $-6581.054^{*}$ & 1342.34 & .000 & -9227.621 & -3934.488 \\
\hline & & $>\mathbf{3 0}$ & $-11236.670^{*}$ & 1396.89 & .000 & -13990.78 & -8482.557 \\
\hline & 25-27.9 & $>\mathbf{3 0}$ & $-6004.459^{*}$ & 1241.97 & .000 & -8453.151 & -3555.767 \\
\hline & $28-29.9$ & $>\mathbf{3 0}$ & $-4655.615^{*}$ & 1411.47 & .001 & -7438.478 & -1872.752 \\
\hline \multirow[t]{2}{*}{ TNF $\alpha$} & \multirow[t]{2}{*}{ 18.5-22.9 } & $28-29.9$ & $-3.019^{*}$ & 1.525 & .049 & -6.027 & -.01215 \\
\hline & & $>30$ & $-4.204^{*}$ & 1.58 & .009 & -7.336 & -1.0717 \\
\hline
\end{tabular}

Note: $\mathrm{ns}=$ non-significant, $\mathrm{p}<0.05:{ }^{*}, \mathrm{p}<0.01:^{* *}, \mathrm{p}<0.001:^{* * *} ; \#$ Frequency of Consumption 
As seen in table 10 (a) those subjects who exercised more had a tendency of increased BMI $(26.4 \mathrm{~kg} / \mathrm{m} 2$ to 27.7 $\mathrm{kg} / \mathrm{m} 2$ ), hs-CRP and had higher counts of Bifidobacteria although this increase was not statistically significant. A non significant reduction in leptin and TNF $\alpha$ was observed with increase in physical activity levels. Post Hoc analysis revealed a significant increase from $2.37 \mathrm{mg} / \mathrm{lto} 3.86 \mathrm{mg} / \mathrm{l}$ in hs-CRP levels in the subjects who exercised for less than 200 MET minutes per week to greater than 2000 MET minutes. Fecal Bifidobacteria decreased from 5.63 $\log _{10} \mathrm{CFU} / \mathrm{g}$ to $5.45 \log _{10} \mathrm{CFU} / \mathrm{gas}$ PAL levels increased from $600 \mathrm{MET}$ minutes to $2000 \mathrm{MET}$ minutes. (Table $10(\mathrm{~b}))$

Table 10:- (a) ANOVA to study the effect of degree of physical activity based on MET minutes on the BMI, Bifidobacteria colonization, inflammatory markers and CBC of subjects under study

\begin{tabular}{|c|c|c|c|c|c|}
\hline MET minutes & $\begin{array}{l}\text { BMI } \\
(n=211)\end{array}$ & $\begin{array}{l}\text { Fecal } \\
\text { Bifidobacteria } \\
\log _{10}(\mathrm{CFU} / \mathrm{g}) \\
\mathrm{n}=42\end{array}$ & $\begin{array}{l}\text { hs-CRP } \\
\text { (mg/l) } \\
\text { n=211 }\end{array}$ & $\begin{array}{l}\text { LEPTIN } \\
\text { (pg/ml) } \\
\text { n=209 }\end{array}$ & $\begin{array}{l}\text { TNF } \alpha \\
(\mathbf{p g} / \mathbf{m l}) \\
\mathbf{n}=211\end{array}$ \\
\hline $0-200$ & $26.40 \pm 4.40$ & $5.63 \pm 0.48$ & $2.37 \pm 2.71$ & $10596.33 \pm 6648.21$ & $7.71 \pm 12.57$ \\
\hline 201-600 & $27.21 \pm 3.52$ & $5.83 \pm 0.24$ & $2.07 \pm 1.79$ & $9614.69 \pm 6722.37$ & $5.58 \pm 3.01$ \\
\hline 601-1000 & $26.71 \pm 3.51$ & $5.59 \pm 0.33$ & $2.96 \pm 3.05$ & $1052.82 \pm 8488.22$ & $5.57 \pm 2.66$ \\
\hline 1001-2000 & $27.70 \pm 4.21$ & $5.45 \pm 0.36$ & $2.78 \pm 2.54$ & $1196.02 \pm 6154.95$ & $5.83 \pm 3.31$ \\
\hline$>2000$ & $27.75 \pm 6.17$ & $5.67 \pm 0.33$ & $3.86 \pm 2.92$ & $1129.89 \pm 9724.44$ & $4.66 \pm 4.15$ \\
\hline $\begin{array}{l}\text { ANOVA } \\
\text { (p value) }\end{array}$ & $\begin{array}{l}0.787^{\mathrm{NS}} \\
(\mathbf{0 . 5 3 5})\end{array}$ & $\begin{array}{l}1.203^{\mathrm{NS}} \\
(0.326)\end{array}$ & $\begin{array}{l}1.52^{\mathrm{NS}} \\
(0.195)\end{array}$ & $\begin{array}{l}\mathbf{0 . 5 8 8}^{\mathrm{NS}} \\
(\mathbf{0 . 6 7 2})\end{array}$ & $\begin{array}{l}0.978^{N S} \\
(0.421)\end{array}$ \\
\hline
\end{tabular}

(b)

\begin{tabular}{|c|c|c|c|c|c|c|c|}
\hline \multicolumn{8}{|c|}{ Post Hoc: LSD Test } \\
\hline \multirow{2}{*}{$\begin{array}{l}\text { Dependent } \\
\text { Variable }\end{array}$} & \multirow{2}{*}{$\begin{array}{l}\text { MET } \\
\text { Range }\end{array}$} & \multirow{2}{*}{$\begin{array}{l}\text { MET } \\
\text { Range }\end{array}$} & \multirow{2}{*}{$\begin{array}{l}\text { Mean } \\
\text { Difference }\end{array}$} & \multirow[t]{2}{*}{ Std. Error } & \multirow[t]{2}{*}{ Sig. } & \multicolumn{2}{|c|}{ 95\% Confidence Interval } \\
\hline & & & & & & Lower Bound & Upper Bound \\
\hline Hs-CRP & $\begin{array}{l}201- \\
600\end{array}$ & $>2000$ & $-1.78^{*}$ & .896 & .047 & -3.555 & -.0199 \\
\hline $\begin{array}{l}\text { Fecal } \\
\text { Bifidobacteria }\end{array}$ & $\begin{array}{l}201- \\
600\end{array}$ & $\begin{array}{l}1001- \\
2000\end{array}$ & $.3767^{*}$ & .18448962 & .048 & .0029764 & .7505994 \\
\hline
\end{tabular}

Increase in the intake of antioxidant rich foods did not show any significant difference on the inflammatory markers and Bifidobacteria counts. (Table 11)

Table 11:- ANOVA to study the effect of intake of antioxidants rich foods on various parameters of obese and non obese subjects.

\begin{tabular}{|c|c|c|c|c|c|c|c|}
\hline $\begin{array}{l}\text { Antioxidant } \\
\text { rich foods } \\
\text { Consumption } \\
\text { Score }\end{array}$ & $\begin{array}{l}\text { BMI } \\
\text { n=211 }\end{array}$ & $\begin{array}{l}\text { MET } \\
\mathrm{n}=\mathbf{2 1 1}\end{array}$ & $\begin{array}{l}\text { Prebiotic } \\
\text { Consumpti } \\
\text { on } \\
n=211\end{array}$ & $\begin{array}{l}\text { Bifidobacteri } \\
\text { a } \\
\log _{10}(\text { CFU/g) }\end{array}$ & $\begin{array}{l}\mathrm{hs}-\mathrm{CRP} \\
(\mathrm{mg} / \mathrm{L}) \\
\mathrm{n}=211\end{array}$ & $\begin{array}{l}\text { Leptin } \\
(\mathbf{p g} / \mathbf{m l}) \\
\mathbf{n = 2 0 9}\end{array}$ & $\begin{array}{l}\text { TNFa } \\
(\mathbf{p g} / \mathbf{m L}) \\
\mathbf{n = 2 1 1}\end{array}$ \\
\hline $22-35$ & $26.32 \pm 3.30$ & $814.81 \pm 627.58$ & $32.24 \pm 7.58$ & $5.44 \pm 0.10$ & $2.19 \pm 2.13$ & $11368.96 \pm 7311.98$ & $5.44 \pm 3.10$ \\
\hline 36-50 & $26.68 \pm 3.95$ & $624.48 \pm 638.79$ & $31.23 \pm 5.02$ & $5.63 \pm 0.40$ & $2.57 \pm 2.59$ & $10265.95 \pm 6492.25$ & $6.25 \pm 8.68$ \\
\hline $51-71$ & $28.05 \pm 4.48$ & $752.45 \pm 699.00$ & $32.65 \pm 7.56$ & $5.76 \pm 0.27$ & $2.79 \pm 2.83$ & $11268.75 \pm 8922.28$ & $6.41 \pm 3.09$ \\
\hline $\begin{array}{l}\text { ANOVA } \\
\text { (p value) }\end{array}$ & $\begin{array}{l}2.390^{\mathrm{NS}} \\
0.094\end{array}$ & $\begin{array}{l}1.250^{\mathrm{NS}} \\
0.289\end{array}$ & $\begin{array}{l}1.1333^{\mathrm{NS}} \\
0.324\end{array}$ & $\begin{array}{l}0.924^{N S} \\
0.405\end{array}$ & $\begin{array}{l}0.39^{\mathrm{NS}} \\
0.674\end{array}$ & $\begin{array}{l}0.479^{N S} \\
0.620\end{array}$ & $\begin{array}{l}0.32^{\text {NS }} \\
0.721\end{array}$ \\
\hline
\end{tabular}


Table 12:- (a) ANOVA to study the effect of increasing order of hs-CRP on various parameters of obese and nonobese subjects.

\begin{tabular}{|c|c|c|c|c|c|c|c|}
\hline $\begin{array}{l}\text { hs-CRP } \\
\text { (mg/l) }\end{array}$ & $\begin{array}{l}\text { BMI } \\
(n=211)\end{array}$ & $\begin{array}{l}\text { MET } \\
(n=211)\end{array}$ & $\begin{array}{l}\text { \#Prebiotic } \\
\text { rich } \\
\text { foods }(n=211)\end{array}$ & $\begin{array}{l}\text { \#Antioxidant } \\
\text { rich foods } \\
(\mathrm{n}=\mathbf{2 1 1})\end{array}$ & $\begin{array}{l}\text { Bifidobacteria } \\
\log _{10}(\mathrm{CFU} / \mathrm{g})\end{array}$ & $\begin{array}{l}\text { LEPTIN } \\
(\mathrm{pg} / \mathrm{ml}) \\
(\mathrm{n}=209)\end{array}$ & $\begin{array}{l}\text { TNFa } \\
(\mathbf{p g} / \mathbf{m l}) \\
(\mathbf{n}=211)\end{array}$ \\
\hline Normal & $25.39 \pm 3.40$ & $527.03 \pm 521.24$ & $31.00 \pm 6.51$ & $44.45 \pm 9.02$ & $5.90 \pm 0.29$ & $8436.06 \pm 6162.41$ & $5.68 \pm 7.50$ \\
\hline $\begin{array}{l}\text { At moderate } \\
\text { risk }\end{array}$ & $26.88 \pm 4.08$ & $724.16 \pm 731.551$ & $32.49 \pm 6.00$ & $46.20 \pm 10.52$ & $5.40 \pm 0.38$ & $9951.26 \pm 6500.92$ & $5.65 \pm 2.98$ \\
\hline At high risk & $28.78 \pm 3.98$ & $781.22 \pm 676.62$ & $31.54 \pm 5.27$ & $44.91 \pm 9.41$ & $5.63 \pm 0.32$ & $13685.52 \pm 7920.17$ & $7.36 \pm 9.81$ \\
\hline $\begin{array}{l}\text { ANOVA } \\
\text { (p value) }\end{array}$ & $\begin{array}{l}\text { 13.834**** } \\
(0.000)\end{array}$ & $\begin{array}{l}\text { 3.043* } \\
(0.050)\end{array}$ & $\begin{array}{l}1^{1.133}{ }^{\mathrm{NS}} \\
(\mathbf{0 . 3 2 4})\end{array}$ & $\begin{array}{l}0^{0.442}{ }^{\mathrm{NS}} \\
(0.644)\end{array}$ & $\begin{array}{l}5.88 * * \\
(0.006)\end{array}$ & $\begin{array}{l}10.708 * * * * \\
(\mathbf{p}-0.000)\end{array}$ & $\begin{array}{l}1.237^{\mathrm{NS}} \\
(0.292)\end{array}$ \\
\hline
\end{tabular}

Note: ns=non-significant, $\mathrm{p}<0.05: *, \mathrm{p}<0.01:^{* *}, \mathrm{p}<0.001: * * * ; \#$ Frequency of Consumption

(b) Post Hoc; LSD test to study the effect of increasing order of hs-CRP on various parameters of obese and non-

obese subjects.

\begin{tabular}{|c|c|c|c|c|c|c|c|}
\hline \multicolumn{8}{|c|}{ Post Hoc; LSD Test } \\
\hline \multirow[t]{2}{*}{$\begin{array}{l}\text { Dependent } \\
\text { Variable }\end{array}$} & \multirow[t]{2}{*}{ hs-CRP Range } & \multirow[t]{2}{*}{ hs-CRP Range } & \multirow[t]{2}{*}{$\begin{array}{l}\text { Mean } \\
\text { Difference }\end{array}$} & \multirow[t]{2}{*}{$\begin{array}{l}\text { Std. } \\
\text { Error }\end{array}$} & \multirow[t]{2}{*}{ Sig. } & \multicolumn{2}{|c|}{ Confidence } \\
\hline & & & & & & $\begin{array}{l}\text { Lower } \\
\text { Bound } \\
\end{array}$ & $\begin{array}{l}\text { Upper } \\
\text { Bound } \\
\end{array}$ \\
\hline \multirow[t]{6}{*}{ BMI } & \multirow[t]{2}{*}{ Normal } & Moderate Risk & $-1.493^{*}$ & .6383 & .020 & -2.752 & -.2350 \\
\hline & & High Risk & $-3.393^{*}$ & .6456 & .000 & -4.666 & -2.125 \\
\hline & \multirow[t]{2}{*}{ Moderate Risk } & Normal & $1.4935^{*}$ & .6383 & .020 & .2350 & 2.720 \\
\hline & & High Risk & $-1.899^{*}$ & .654 & .004 & -3.189 & -.609 \\
\hline & \multirow[t]{2}{*}{ High Risk } & Normal Level & $3.3931^{*}$ & .645 & .000 & 2.120 & 4.66 \\
\hline & & Moderate Risk & $1.8999^{*}$ & .6543 & .004 & .609 & 3.18 \\
\hline MET & Normal & High Risk & $-254.197^{*}$ & 109.05 & .021 & -469.18 & -39.21 \\
\hline \multirow[t]{2}{*}{ Leptin } & \multirow[t]{2}{*}{ High Risk } & Non-obese & $5249.46^{*}$ & 1159.8 & .000 & 2962.831 & 7536.09 \\
\hline & & Moderate Risk & $3734.261^{*}$ & 1183.87 & .002 & 1400.187 & 6068.33 \\
\hline
\end{tabular}

Table 13:- Pearsons correlation amongst various parameters of the subjects under study

\begin{tabular}{|c|c|c|c|c|c|c|c|c|c|}
\hline \multicolumn{2}{|l|}{ Parameters and $\mathbf{N}$} & $\begin{array}{l}\text { BMI } \\
(211)\end{array}$ & $\begin{array}{l}\text { PAL }^{@} \\
(\mathbf{2 1 1})\end{array}$ & $\begin{array}{l}\text { Pre-pro } \\
\text { intake } \\
(211)\end{array}$ & $\begin{array}{l}\text { Anti- } \\
\text { oxidant }^{\$} \\
(211)\end{array}$ & $\begin{array}{l}\text { Hs- } \\
\text { CRP } \\
(211)\end{array}$ & $\begin{array}{l}\text { Leptin } \\
\text { (209) }\end{array}$ & $\begin{array}{l}\text { TNFa } \\
(211)\end{array}$ & $\begin{array}{l}\text { Bifido- } \\
\text { bacteria count } \\
(42)\end{array}$ \\
\hline BMI & $\mathbf{R}$ & 1 & .062 & .038 & .133 & $.325^{* *}$ & $.534^{* *}$ & $.191^{* *}$ & -.048 \\
\hline PAL & $\mathbf{R}$ & .062 & 1 & .131 & .098 & .095 & .044 & -.080 & -.135 \\
\hline Pre pro intake ${ }^{\#}$ & $\mathbf{R}$ & .038 & .131 & 1 & $.209^{3 * *}$ & -.042 & -.018 & -.046 & -.183 \\
\hline Antioxidant $^{\$}$ & $\mathbf{R}$ & .133 & .098 & $.209^{* * *}$ & $\mathbf{1}$ & .031 & .014 & -.031 & .060 \\
\hline Hs-CRP & $\mathbf{R}$ & $.325^{* * *}$ & .095 & -.042 & .031 & 1 & $.339^{* * *}$ & .095 & -.168 \\
\hline Leptin & $\mathbf{R}$ & $.534^{* * *}$ & .044 & -.018 & .014 & $.339^{* * *}$ & 1 & .109 & .038 \\
\hline TNFa & $\mathbf{R}$ & $.191^{* * *}$ & -.080 & -.046 & -.031 & .095 & .109 & 1 & .106 \\
\hline $\begin{array}{l}\text { Bifidobacteria } \\
\text { counts }\end{array}$ & $\mathbf{R}$ & -.048 & -.135 & -.183 & .060 & -.168 & .038 & .106 & 1 \\
\hline
\end{tabular}

$\mathrm{p}<0.01$ ** $^{*}$-\$-Consumption of antioxidant rich food; \#- Consumption of Prebiotic rich food ; @-physical activity level 
As seen in table $14(\mathrm{a}-\mathrm{f})$ Step wise regression analysis revealed leptin $\left(\mathrm{r}^{2}=0.631\right)$ followed by hs-CRP $\left(\mathrm{r}^{2}=0.641\right)$ as the important predictors for obesity.

Table 14:- Regression analysis to determine the factors affecting obesity.

(a)

\begin{tabular}{|c|c|c|c|}
\hline \multicolumn{3}{|c|}{ Descriptive Statistics } \\
\hline BMI & Mean & Std. Deviation & $\mathrm{N}$ \\
\hline hs-CRP & 28.558 & 3.0314102 & 159 \\
\hline LEPTIN & 2.820 & 2.6696187 & 159 \\
\hline TNFa & 11961.656 & 7243.868236 & 159 \\
\hline PLATELET_COUNT & 6.9307 & 8.214 & 159 \\
\hline
\end{tabular}

(b)

\begin{tabular}{|l|c|c|c|}
\hline \multicolumn{3}{|c|}{ Variables Entered/Removed $^{\text {a }}$} \\
\hline Model & Variables Entered & Variables Removed & Method $^{\text {LEPTIN }}$ \\
& $\cdot$ & $\cdot$ & $\begin{array}{c}\text { Stepwise (Criteria: Probability-of-F-to-enter <= .050, } \\
\text { Probability-of-F-to-remove >= .100). }\end{array}$ \\
\hline \multicolumn{2}{|c|}{ a. Dependent Variable: BMI } & \\
\hline
\end{tabular}

(c)

\begin{tabular}{|c|c|c|c|c|}
\hline \multicolumn{5}{|c|}{ Model Summary $^{\mathbf{b}}$} \\
\hline Model & $\mathrm{R}$ & R Square & Adjusted R Square & Std. Error of the Estimate \\
\hline 1 & $.410^{\mathrm{a}}$ & $\mathbf{. 1 6 8}$ & $\mathbf{. 1 6 3}$ & 2.7739672 \\
\hline \multicolumn{4}{|c|}{ a. Predictors: (Constant), LEPTIN } \\
\hline
\end{tabular}

(d)

\begin{tabular}{|c|c|c|c|c|c|c|}
\hline \multicolumn{7}{|c|}{ ANOVA $^{b}$} \\
\hline \multicolumn{2}{|c|}{ Model } & Sum of Squares & Df & Mean Square & $\mathbf{F}$ & Sig. \\
\hline \multirow[t]{3}{*}{1} & Regression & 243.834 & 1 & 243.834 & 31.688 & $.000^{\mathrm{a}}$ \\
\hline & Residual & 1208.098 & 157 & 7.695 & & \\
\hline & Total & 1451.933 & 158 & & & \\
\hline \multicolumn{4}{|c|}{ a. Predictors: (Constant), LEPTIN } & & & \\
\hline & Dependent $\mathbf{V}$ & ble: BMI & & & & \\
\hline
\end{tabular}

(e)

\begin{tabular}{|c|c|c|c|c|c|c|}
\hline \multicolumn{7}{|c|}{ Coefficients $^{\mathrm{a}}$} \\
\hline \multicolumn{2}{|c|}{ Model } & \multicolumn{2}{|c|}{ Unstandardized Coefficients } & \multirow{2}{*}{$\begin{array}{c}\begin{array}{c}\text { Standardized } \\
\text { Coefficients }\end{array} \\
\text { Beta } \\
\end{array}$} & \multirow[t]{2}{*}{$\mathbf{t}$} & \multirow[t]{2}{*}{ Sig. } \\
\hline & & B & Std. Error & & & \\
\hline \multirow[t]{2}{*}{1} & (Constant) & 26.508 & .426 & & 62.273 & .000 \\
\hline & LEPTIN & .000 & .000 & .410 & 5.629 & .000 \\
\hline \multicolumn{3}{|c|}{ a. Dependent Variable: BMI } & & & & \\
\hline
\end{tabular}

(f)

\begin{tabular}{|c|c|c|c|c|c|c|}
\hline \multicolumn{7}{|c|}{ Excluded Variables $^{b}$} \\
\hline \multicolumn{2}{|r|}{ Model } & Beta In & $\mathrm{T}$ & Sig. & $\begin{array}{c}\text { Partial } \\
\text { Correlation }\end{array}$ & $\begin{array}{l}\text { Collinearity } \\
\text { Statistics }\end{array}$ \\
\hline \multirow{3}{*}{1} & HS-CRP & $112^{\mathrm{a}}$ & 1468 & 144 & 117 & $\frac{\text { Tolerance }}{904}$ \\
\hline & $\mathrm{TNF} \alpha$ & $.083^{\mathrm{a}}$ & 1.142 & .255 & .091 & 997 \\
\hline & PLATELET_COUNT & $.112^{\mathrm{a}}$ & 1.538 & .126 & .122 & .986 \\
\hline \multicolumn{4}{|c|}{ a. Predictors in the Model: (Constant), LEPTIN } & & & \\
\hline b. D & lent Variable: BMI & & & & & \\
\hline
\end{tabular}




\section{Discussion:-}

The obesity epidemic has led us to evaluate the alternative mechanisms involved in high rates of obesity related co morbidities. This includes the role of physical activity, systemic inflammation, Gut health, dietary intakes of prebiotic and antioxidant rich foods. Reframing obesity as an inflammatory condition has had a wide impact on our conceptualization of obesity and associated non communicable diseases. The present study was conducted with the primary objective to determine the various factors contributing to levels of inflammatory markers in obese individuals and compare them with non obese subjects.

Results of present study indicated that the obese subjects enrolled for the study belonged to grade I obesity and no significant difference was observed in their BMI values with respect to their activity levels.

The mean values for anti-inflammatory markers in terms of hs-CRP, TNF $\alpha$ and leptin were significantly higher in obese subjects as compared to non-obese subjects. This can be justified as they are all obese and adipose tissue is a source for the production and release of cytokines, which stimulates the synthesis of CRP by the liver. A study conducted by Farhangi et al. (2013) reported that obese subjects had significantly higher hs-CRP and IL-6 concentrations $(p<0.05)$ than their non obese counterparts. Similar results were observed by Barter P et al. 2007, who reported significantly higher values of $3.0 \mathrm{mg} / \mathrm{l}$ in obese individuals as compared to $2.0 \mathrm{mg} / \mathrm{l}$ in non obese individuals. Similar trend of hs-CRP was also found in Indian obese individuals (2.86 mg/l) (Reddy et al, 2013). Higher TNF $\alpha$ levels were observed in obese subjects by Tzanavari T et al (2010).

A progressive increase in serum Leptin concentration was observed with an increase in BMI. Also significant difference between Leptin concentrations in either gender was found in non obese and obese subjects. Paul $\mathrm{R}$ et al in 2011 reported the mean values for leptin were $2.6+/-1.5 \mathrm{etag} / \mathrm{ml}$ in men, and $17.3+/-10.2 \mathrm{etag} / \mathrm{ml}$ in women with normal BMI whereas in obese subjects the mean values of leptin were $21.3+/-14.2 \mathrm{etag} / \mathrm{ml}$ in men, and $48.21+/$ $21.2 \mathrm{etag} / \mathrm{ml}$ in women $(\mathrm{p}<0.001)$. Significant associations has also been reported between BMI and serum leptin levels, where the median serum leptin concentration in overweight subjects was $19.6(2.0-60.0 \mathrm{ng} / \mathrm{ml})$ compared with 9.0 (range 1.0-30.0 ng/ml) in the control subjects ( $<$ 0.001) (Tungtrongchitr R et al, 2000).

Aging is associated with a decrease in all major components of Total Energy Expenditure, including resting metabolic rate, thermic effect of food, hormonal changes and physical activity, leading to an increase in BMI (Villareal D et al 2005; Mungreiphy N et al, 2011). However, rejecting the belief that BMI is associated with age and gender of the subjects, the present study depicted a contrary picture. No significant association was found between age of the subjects and the inflammatory markers, BMI and physical activity. However, a 24 year longitudinal study documented that, mean BMI increased from 24.1 to 25.5 for men and from 23.1 to 24.3 for women during the 24-year study period. Overall, BMI increased by $1.4 \mathrm{~kg} / \mathrm{m}^{2}$ in men and by $1.2 \mathrm{~kg} / \mathrm{m}^{2}$ in women during the course of the study period. Similar results of increasing BMI with age have also been reported in studies conducted for a longitudinal period of 10 years in US on adults from 25- 74 years and 5 years in Sweden on adults between 18- 80 years (Williamson D, 1990; Emrani F et al, 2013). The contrary results observed in the present study can be attributed to an unequal sample size in all the age groups.

The present study depicted a non-significant association between age and inflammatory markers. A few studies have also shown similar results, which do not support an increase in the production of pro inflammatory cytokines IL-1 $\beta$ and IL-6 with aging when health and nutritional status are maintained (Ahluwalia N et al, 2001). Data from a study conducted by Beharka A et al, have also documented that in healthy subjects, increased production of IL-6 is not a normal consequence of aging.

The expansion of the adipose tissue leads to the altered production of proinflammatory molecules and results in lowgrade inflammation. The increase in hs-CRP levels indicates a state of low-grade inflammation in the obese group (Dev and Marcus, 2012). Also high circulating leptin in body is directly associated to hs-CRP levels (Ukkola O, Kesäniemi 2007; Hussain D, 2012). The present study had also depicted a similar trend with a higher level of both leptin and hs-CRP more so in females than in males. Similar results have also been supported by Delongui $\mathrm{F}$ et al in his study conducted on healthy adults from Brazil depicting a higher level of hs-CRP in females (1.89mg/l) than in males $(0.89 \mathrm{mg} / \mathrm{l})$. Thus it can be concluded that hs -CRP has shown a strong positive correlation with serum leptin levels, gender and BMI. 
The results showed an increasing trend in hs-CRP levels in association with the increasing MET levels. As the MET minutes indicating physical activity levels increased from 200 to 2000, there was an increase in hs-CRP levels from 2.37 to $3.86 \mathrm{mg} / \mathrm{L}$. This finding is in line with an earlier observation in subjects which concluded that although physical fitness was significantly associated with circulating CRP level in a cross-sectional study, increasing fitness did not proportionally decrease circulating CRP level as greatest reduction in hs-CRP was found in subjects with mildly increased fitness $(\mathrm{n}=159 ; 0.35$ to $0.21 \mathrm{mg} / \mathrm{L} ; \mathrm{P}<.0001)$, but not in those with moderately to highly increased fitness ( $\mathrm{n}=113 ; 0.36$ to $0.28 \mathrm{mg} / \mathrm{L} ; \mathrm{P}=.03$ ) (Nakajima $\mathrm{K}$ et al, 2008), suggesting that, high physical activity levels are not necessarily associated with reduction in inflammatory markers in obese individuals. In the present study increased inflammatory markers in the moderately active subjects can be attributed to the fact that the blood of the subjects was drawn soon after the activity was performed. Studies have reported that the inflammatory markers remain high upto 48 hours of performing moderately physical activity.

Interestingly, the present study has also shown that there is no association between hs-CRP levels and increased frequency of intake of antioxidant rich foods, as proven in some other studies too. A study on consumption of Dietary Antioxidants by 150 subjects with coronary heart disease showed no effect on hs-CRP levels ( $\mathrm{p}=0.17)$ even after consumption of high antioxidant rich food (Niknam M, 2015). Similar results were observed by Oliveira A et al in a study where, on increasing the consumption of $100 \mathrm{gms}$ of fruit and vegetable showed no change in the hsCRP levels in both genders. Thus it can be assumed that obesity and risk of NCD's cannot be reduced only by consumption of antioxidant rich foods.

\section{References:-}

1. Ahluwalia N, Mastro AM, Ball R, Miles MP, Rajendra R and Handte G. Cytokine production by stimulated mononuclear cells did not change with aging in apparently healthy, well-nourished women. Mechanisms of ageing and development, 2001; 122: 1269-1279

2. Al-Emrani F, Stafström M, Björk J, and Per-OlofÖstergren. Pattern of Five-Year Weight Change by Age and Birth Cohorts in a Swedish Adult Population. The Open Public Health Journal, 2013; 6: 42-50

3. Barter P, McPherson YR, Song K, Kesäniemi A, Mahley R, Waeber G, et al. Serum insulin and inflammatory markers in overweight individuals with or without dyslipidemia. J ClinEndocrinolMetab 2007;92:2041-2045

4. Beharka AA, Meydani M, Wu D, Leka LS, Meydani A and Meydani SN. Interleukin-6 production does not increase with age. The journals of gerontology Series A, Biological sciences and medical sciences, 2001; 56: $81-88$

5. Delongui F, Kallaur AP, Oliveira SR, Bonametti AM, Grion CM, Morimoto HK, Simão AN, Magalhães GG, Reiche EM. Serum levels of high sensitive C reactive protein in healthy adults from southern Brazil. J Clin Lab Anal. 2013;27(3):207-10.

6. Dixit VD. Adipose-immune interactions during obesity and caloric restriction: reciprocal mechanisms regulating immunity and health span. J Leukoc Biol. 2008;8:882-92. [PMC free article] [PubMed]

7. Farhangi MA, Keshavarz SA, Eshraghian M, Ostadrahimi A, Saboor-Yaraghi AA. White blood cell count in women: relation to inflammatory biomarkers, haematological profiles, visceral adiposity, and other cardiovascular risk factors. Journal of Health, Population and Nutrition. 2013 Apr 25;31(1):58-64.

8. Hotamisligil GS, Erbay E. Nutrient sensing and inflammation in metabolic diseases. Nat Rev Immunol.2008;8:923-934. [PMC free article] [PubMed]

9. Hussain SD. The correlation between serum high sensitivity c-reactive protein and leptin in reproductive age overweight/obese women in Erbil city. Zanco J. Med. Sci., 2012; 16 (3)

10. Luchsinger JA, Gustafson DR. Adiposity, type 2 diabetes, and Alzheimer's disease. J Alzheimers Dis.2009;16:693-704. [PMC free article] [PubMed]

11. Maier W, Altwegg LA, Corti R, Gay S, Hersberger M, Maly FE, Sütsch G, Roffi M, Neidhart M, Eberli FR, Tanner FC. Inflammatory markers at the site of ruptured plaque in acute myocardial infarction locally increased interleukin-6 and serum amyloid A but decreased C-reactive protein. Circulation. 2005 Mar 22;111(11):135561.

12. Mungreiphy NK, Kapoor S, and Sinha R. Association between BMI, Blood Pressure, and Age: Study among Tangkhul Naga Tribal Males of Northeast India. Journal of Anthropology. 2011; 11(6): 1-6

13. Nakajima K, Kusuhara M, Yonemura A, Ayaori M, Saionji K, Tamai S, Ohsuzu F. Increasing physical fitness does not proportionally decrease circulating C-reactive protein level in men with varying fitness. Metabolism. 2008;57(5):650-7 
14. Niknam M, Paknahad Z, Baghestani A and Hashemi M. Anti-Inflammatory Effects of Dietary Antioxidants in Patients with Coronary Artery Disease. EndocrinolMetabSyndr 2015, 4:4

15. Park HS, Park JY, \& Yu R. Relationship of obesity and visceral adiposity with serum concentrations of CRP, TNF- $\alpha$ and IL-6. Diabetes research and clinical practice, 69(1), (2005). 29-35.

16. Paul RF, Hassan M, Hassan SN, Gillani S, Afzal N, Qayyum I. Effect of body mass index on serum leptin levels. J Ayub Med Coll Abbottabad 2011;23(3)

17. Petersen AMW, \& Pedersen BK. The anti-inflammatory effect of exercise. Journal of applied physiology, 98(4), (2005) 1154-1162.

18. Tungtrongchitr R, Pongpaew P, Phonrat B, Tribunyatkul S, Viroonudomphol D, Supawan V, Jintaridhi P, Lertchavanakul A, Vudhivai N and Schelp FP. Leptin concentration in relation to body mass index (BMI) and hematologicalmeasurements in Thai obese and overweight subjects. Southeast Asian J trop med public health, 2000; 31(4)

19. Tzanavari T, Giannogonas P, Karalis KP. TNF-alpha and obesity. Curr Dir Autoimmun. 2010;11:145-56

20. Ukkola O, Kesäniemi YA. Leptin and high-sensitivity C-reactive protein and their interaction in the metabolic syndrome in middle-aged subjects. Metabolism. 2007 Sep;56(9):1221-7

21. Villareal DT, Apovian CM, Kushner RF, and Klein S. Obesity in older adults: technical review and position statement of the American Society for Nutrition and NAASO, The Obesity Society. Am J ClinNut,r2005;82:923-34

22. Williamson DF, Kahn HS, Remington PL, Anda RF. The 10-year incidence of overweight and major weight gain in US adults. Arch Intern Med. 1990;150(3):665-72.

23. World Health Organization. Global Health Estimates: Deaths by Cause, Age, Sex and Country, $2000-2012$. Geneva, WHO, 2014

24.World Health Organization, Global Physical Activity Questionnaire, 2007

25. Yang H, et al. Obesity accelerates thymic aging. Blood. 2009;114:3803-3812. 